\title{
A NEW THEOREM IN ANALYTIC CONICS.
}

By Alan S. Hawkesworth (Pittsburgh, Pa.)

Adunanza del 14 novembre 1915.

If $P U$ be the normal of any point $P$ on a central conic. And through $P$ chords $P Q, P R$ be drawn at right angles; cutting the conic again in $Q ; R$ respectively. And chord $Q R$ cuts the normal $P U$ in $U$.

Then will the magnitude $P U$ ever bear the same fixed ratio to the semi diameter conjugate to the diameter through $P$, that twice the product of the semi axi of the conic bears to the sum [if an Ellipse], or the difference [if a Hyperbola], of the squares of those semi-axi.

Let $x_{1}, y_{1}$ be the coördinates of the point $P$ on the conic $b^{2} x^{2} \pm a^{2} y^{2}-a^{2} b^{2}=0$. [with the conic's axi for the rectangular axi. When the signs are ambiguous; the upper is that for the Ellipse; the lower for the Hyperbola].

Then the equation of the normal at $P$ is

or

$$
y-y_{1}=\frac{ \pm a^{2} y_{1}}{b^{2} x_{\mathrm{r}}}\left(x-x_{\mathrm{r}}\right)
$$

$$
y=\frac{ \pm a^{2} y_{1}}{b^{2} x_{1}} x \mp \frac{a^{2} y_{1}}{b^{2} x_{1}} x_{1}+\frac{b^{2} x_{1} y_{1}}{b^{2} x_{1}} \text {. }
$$

Now, by a known theorem, if perpendicular chords $P Q, P R$ be drawn throu any point $P$ on any conic; cutting it again in $Q, R$. Then will chord $Q R$ cut the normal of $P$ in a point $U$; fixed for that normal. For convenience, then, let chords $P Q, P R$ be drawn parallel to the axi. When chord $Q U R$, cutting normal $P U$ in $U$, will be a diameter; symmetric to that through $P$. Whereupon the equation of diameter $Q U R$ will be $y_{1} x+x_{1} y=0$ or $y=\frac{-y_{1}}{x_{1}} x$.

Hence

$$
\frac{-y_{1}}{x_{1}} x=\frac{ \pm a^{2} y_{1}}{b^{2} x_{1}} x \mp \frac{a^{2} y_{1}}{b^{2} x_{1}} x_{1}+\frac{b^{2} x_{1} y_{1}}{b^{2} x_{1}}=\frac{y_{1}\left( \pm a^{2} x \mp a^{2} x_{1}+b^{2} x_{1}\right)}{b^{2} x_{1}} \text {. }
$$

Dividing by $\frac{y_{1}}{x_{i}}$ we obtain

$$
-x= \pm \frac{a^{2}}{b^{2}}\left(x-x_{1}\right)+x_{1}
$$


or

$$
\left( \pm a^{2}+b^{2}\right) x=\left( \pm a^{2}-b^{2}\right) x_{1} .
$$

So that the coördinates $x_{2}, y_{2}$ of point $U$, wherein diameter $Q U R$ cuts the normal $P U$, are

and

$$
x_{2}=\left(\frac{ \pm a^{2}-b^{2}}{ \pm a^{2}+b^{2}}\right) x_{\mathrm{I}}
$$

$$
y_{2}=-\left(\frac{ \pm a^{2}-b^{2}}{ \pm a^{2}+b^{2}}\right) y_{1} \text {. }
$$

Hence the magnitude $P U$ must be

$$
\begin{aligned}
& \sqrt{\left(x_{1}-\frac{ \pm a^{2}-b^{2}}{ \pm a^{2}+b^{2}} x_{1}\right)^{2}+\left(y_{1}+\frac{ \pm a^{2}-b^{2}}{ \pm a^{2}+b^{2}} y_{1}\right)^{2}} \\
& =\sqrt{\frac{\left( \pm a^{2} x_{1}+b^{2} x_{1} \mp a^{2} x_{1}+b^{2} x_{1}\right)^{2}+\left( \pm a^{2} y_{1}+b^{2} y_{1} \pm a^{2} y_{1}-b^{2} y_{1}\right)^{2}}{\left( \pm a^{2}+b^{2}\right)^{2}}} \\
& =\frac{2}{\left| \pm a^{2}+b^{2}\right|} \sqrt{b^{4} x_{1}^{2}+a^{4} x_{1}^{2}} \text {. }
\end{aligned}
$$

But the coördinates $x_{3}, y_{3}$ of the semidiameter conjugate to that through $P=x_{5}$, $y_{1}$ being $x_{3}=\mp \frac{a}{b} y_{1} ; y_{3}=\frac{b}{a} x_{1}$. It follows that its magnitude is $\sqrt{\frac{a^{4} y_{1}^{2}+b^{4} x_{1}^{2}}{a^{2} b^{2}}}$ so that the fixed ratio of the magnitude $P U$ of the normal at $P$ is ever to that conjugate semidiameter as $\frac{2 a b}{ \pm a^{2}+b^{2}}$ or $\frac{2 a b}{a^{2} \pm b^{2}}$; the ratio being an absolute value.

Note I. - In the Hyperbola, the triangle of points $P Q R$ can obviously all lie on the conjugate curve.

Note II. - When the asymptotal angle is less that right; then $P U$ lies within the curve. When greater, then externally. While in the Equilateral Hyperbola $a^{2}-b^{2}$ disappears; $P U$ is thus infinite; and parallel to the conjugate semi diameter.

Note III. - In the Parabola chord $P Q$ being drawn perpendicular, and diameter $Q U$ parallel to its axis. It is plain that magnitude $P U$ is bisected by the axis; and $Q U=p$. the Parabola's parameter. So that $\theta$ being the angle in which normal $P U$ cuts the axis, $P U=p \cdot \sec \theta$.

Thus the unknown infinite diameters at infinity, conjugate to those through say $P$ and $P_{\mathrm{I}}$ respectively, must be to each other in the ratio of $\frac{\sec \theta}{\sec \theta_{1}}$ or inversely, as $\frac{\cos \theta}{\cos \theta}$.

Pittsburgh, $14^{\text {th }}$ novembre 1915 .

Alan S. Hawkesworth. 